\title{
A CHARACTERISATION OF THE NORMALISERS OF C*-ALGEBRAS
}

\author{
I. G. TODOROV \\ Department of Pure Mathematics, Queen's University, Belfast BT7 1NN, Northern Ireland \\ e-mail:i.todorov@qub.ac.uk
}

(Received 19 May, 2003; accepted 4 May, 2004)

\begin{abstract}
We characterise the ternary rings of operators possessing a completely isometric representation whose range consists of normalisers or semi-normalisers between the ranges of some ${ }^{*}$-representations of two fixed $\mathrm{C}^{*}$-algebras. We give some corollaries of these results.
\end{abstract}

2000 Mathematics Subject Classification. 46L07, 46L08.

1. Introduction and preliminaries. Normalisers have played a major role in the theory of selfadjoint operator algebras since their introduction by Murray and von Neumann [12]. Their importance in the rapidly growing theory of limit algebras is vital [15]. Normalisers of non-selfadjoint, and more specifically of nest, algebras were introduced in [5] and subsequently studied in the more general setting of reflexive algebras in [8].

There exists a close relation between normalisers and ternary rings of operators (TRO's). Recall that a TRO is a norm closed subspace $\mathcal{U}$ of the space $\mathcal{B}\left(\mathcal{H}_{1}, \mathcal{H}_{2}\right)$ of all bounded linear operators from a Hilbert space $\mathcal{H}_{1}$ into a Hilbert space $\mathcal{H}_{2}$ such that $T S^{*} R \in \mathcal{U}$ whenever $T, S, R \in \mathcal{U}$. Let $\mathcal{A}$ and $\mathcal{B}$ be norm closed (in general, nonselfadjoint) operator algebras acting on $\mathcal{H}_{1}$ and $\mathcal{H}_{2}$, respectively, and $T \in \mathcal{B}\left(\mathcal{H}_{1}, \mathcal{H}_{2}\right)$ a normaliser of $\mathcal{B}$ into $\mathcal{A}$; that is, an operator satisfying the relations

$$
T^{*} \mathcal{B} T \subseteq \mathcal{A} \text { and } T \mathcal{A} T^{*} \subseteq \mathcal{B}
$$

It is easy to see that the TRO $\mathcal{U} \subseteq \mathcal{B}\left(\mathcal{H}_{1}, \mathcal{H}_{2}\right)$ that is generated by $T$ consists of normalisers of $\mathcal{B}$ into $\mathcal{A}$; thus each normaliser of $\mathcal{B}$ into $\mathcal{A}$ belongs to a TRO each of whose elements normalises $\mathcal{B}$ into $\mathcal{A}$. As a matter of fact, if $\mathcal{A}$ and $\mathcal{B}$ are reflexive algebras, this holds also for the semi-normalisers of $\mathcal{B}$ into $\mathcal{A}[\mathbf{8}]$; that is, the operators $T \in \mathcal{B}\left(\mathcal{H}_{1}, \mathcal{H}_{2}\right)$ satisfying only the first of the relations (1).

Both operator algebras and TRO's can be viewed abstractly in the setting of Operator Space Theory (see e.g. [1], [6] and [7]). Suppose that $A$ and $B$ are abstract operator algebras. It is natural to ask for an abstract characterisation of the TRO's $X$ possessing a completely isometric representation as a space of normalisers between (the ranges of) some completely isometric representations of $A$ and $B$. In the present note we provide such a characterisation in the case in which $A$ and $B$ are $C^{*}$-algebras. Concrete representations of abstract operator spaces, algebras, modules, etc. are of basic importance in Operator Space Theory. See [1] and [6]. Our results establish such representations in a new situation and point out a direction to consider normalisers of 
operator algebras independently of the underlying Hilbert spaces. They extend a well known representation of the Morita context of two strongly Morita equivalent (in the sense of Rieffel [16]) $C^{*}$-algebras. (We refer the reader to [2] for the categorical aspects of Morita equivalence of $\mathrm{C}^{*}$-algebras.) Recall that if $A$ and $B$ are strongly Morita equivalent $\mathrm{C}^{*}$-algebras then their imprimitivity bimodule, say $X$, is a TRO, and $A, B$ and $X$ can be concretely and faithfully represented in such a way that the linear span of $X^{*} X$ is dense in $A$ and the linear span of $X X^{*}$ is dense in $B$. (See [3] for a more general representation of Morita contexts of non-selfadjoint operator algebras.) In that case $X$ is a "full" space of normalisers of $B$ into $A$. In our setting, $A$ and $B$ need not be Morita equivalent; we characterise the normalising TRO's for which $X^{*} B X$ and $X A X^{*}$ do not necessarily span dense subsets of $A$ and $B$.

The question we study is closely related to the notion of a Hilbert module over a $\mathrm{C}^{*}$-algebra. As corollaries, we provide an intrinsic characterisation of the sub-TRO's of the Hilbert modules over a given $\mathrm{C}^{*}$-algebra and a representation for a class of positive definite maps. We also discuss weak* versions of our main results.

We briefly review the concept of a Hilbert (bi)module. Let $A$ be a $\mathrm{C}^{*}$-algebra. A complex linear space $Z$ is called a right pre-Hilbert $A$-module if $Z$ is a right $A$-module and an inner product $(\cdot, \cdot)_{A}: Z \times Z \rightarrow A$ is given such that, for each $z, z_{1}, z_{2} \in Z$ and $a \in A$, the following identities hold:

(i) $\left(z_{1}, z_{2} \cdot a\right)_{A}=\left(z_{1}, z_{2}\right)_{A} a$;

(ii) $\left(z_{1}, z_{2}\right)_{A}^{*}=\left(z_{2}, z_{1}\right)_{A}$;

(iii) $(z, z)_{A} \geq 0$ and $(z, z)_{A}=0$ if and only if $z=0$.

Left pre-Hilbert modules are defined similarly. If $A$ and $B$ are $\mathrm{C}^{*}$-algebras, the space $Z$ is called a pre-Hilbert $B, A$-bimodule [4], if $Z$ is a right pre-Hilbert $A$-module, a left pre-Hilbert $B$-module and the identity

(iv) $\left(z_{1}, z_{2}\right)_{B} \cdot z_{3}=z_{1} \cdot\left(z_{2}, z_{3}\right)_{A}$

holds for each $z_{1}, z_{2}, z_{3} \in Z$. Pre-Hilbert (bi)modules, which are complete with respect to the norm $\|x\|=\sqrt{\left\|(x, x)_{A}\right\|}$ are called Hilbert (bi)modules.

If $\mathcal{U} \subseteq \mathcal{B}\left(\mathcal{H}_{1}, \mathcal{H}_{2}\right)$, let $M_{n}(\mathcal{U}) \subseteq \mathcal{B}\left(\mathcal{H}_{1}^{n}, \mathcal{H}_{2}^{n}\right)$ denote the space of all $n$ by $n$ matrices with entries in $\mathcal{U}$. If $\mathcal{U} \subseteq \mathcal{B}\left(\mathcal{H}_{1}, \mathcal{H}_{2}\right)$ and $\mathcal{V} \subseteq \mathcal{B}\left(\mathcal{K}_{1}, \mathcal{K}_{2}\right)$ are norm closed spaces, a linear map $\varphi: \mathcal{U} \rightarrow \mathcal{V}$ is called a complete isometry if the map $\varphi_{n}: M_{n}(\mathcal{U}) \rightarrow M_{n}(\mathcal{V})$, given by $\varphi_{n}\left(\left(x_{i j}\right)\right)=\left(\varphi\left(x_{i j}\right)\right)$, is an isometry for each $n \in \mathbb{N}$.

Suppose that $\mathcal{U} \subseteq \mathcal{B}\left(\mathcal{H}_{1}, \mathcal{H}_{2}\right)$ and $\mathcal{V} \subseteq \mathcal{B}\left(\mathcal{K}_{1}, \mathcal{K}_{2}\right)$ are TRO's. A linear map $\varphi$ : $\mathcal{U} \rightarrow \mathcal{V}$ will be called a ternary monomorphism if $\varphi$ is injective and $\varphi\left(T S^{*} R\right)=$ $\varphi(T) \varphi(S)^{*} \varphi(R)$, for each $T, S, R \in \mathcal{U} ; \varphi$ is a ternary isomorphism, if it is a surjective ternary monomorphism. Ternary isomorphisms between TRO's are complete isometries and vice versa [7]; hence we can speak about abstract TRO's in the sense of Operator Space Theory [9]. Alternatively, abstract TRO's can be defined axiomatically [18], [19]. Following [9] and [17] however, we shall not make a strict distinction between abstract and concrete TRO's, just as one does in the case of $\mathrm{C}^{*}$-algebras.

Let $\left(Z,(\cdot, \cdot)_{A}\right)$ be a Hilbert module over a $C^{*}$-algebra $A$. By [11] and [19], there exists an isometry $\varphi: Z \rightarrow \mathcal{B}(\mathcal{H}, \mathcal{K})$ and a faithful ${ }^{*}$-representation $\pi: A \rightarrow \mathcal{B}(\mathcal{H})$ such that $\pi\left(\left(z_{1}, z_{2}\right)_{A}\right)=\varphi\left(z_{1}\right)^{*} \varphi\left(z_{2}\right)$ and $\varphi(z \cdot a)=\varphi(z) \pi(a)$. It is easy to see that $\varphi(Z)$ is a TRO and that any two such representations of $Z$ are ternarily isomorphic. Hence, $Z$ can be viewed as a TRO itself, its ternary product being $z_{1} z_{2}^{*} z_{3}=z_{1} \cdot\left(z_{2}, z_{3}\right)_{A}$. (We refer the reader to Section 2 of [17] for a detailed account of the relationship between TRO's and Hilbert modules.) If $Z$ is a Hilbert bimodule, then condition (iv) guarantees that the ternary structures arising from the two (different) $\mathrm{C}^{*}$-algebras coincide. 
2. Characterisation results. Let $A, B$ be unital $\mathrm{C}^{*}$-algebras and $X$ a complex linear space. Suppose that the map $r: X \times B \times X \rightarrow A$ is linear on the first and the second variable and conjugate linear on the third, or linear on the second and the third variable and conjugate linear on the first. (We shall call such maps sesquilinear, pointing out in addition on which variable we have conjugate linearity.) Call $r$ positive definite if $\sum_{i, j=1}^{n} a_{i}^{*} r\left(x_{i}, b_{i}^{*} b_{j}, x_{j}\right) a_{i} \geq 0$ in $A$, for all $x_{i} \in X, a_{i} \in A, b_{i} \in B, i=1,2, \ldots, n$. By Proposition 6.1 of [13], the map $r$ is positive definite if and only if for any $x_{1}$, $x_{2}, \ldots, x_{n} \in X$ and each positive matrix $\left(b_{i j}\right)_{i j} \in M_{n}(B)$ the matrix $\left(r\left(x_{i}, b_{i j}, x_{j}\right)\right)_{i j} \in$ $M_{n}(A)$ is positive. Call $r$ weakly positive definite, if $\sum_{i, j=1}^{n} r\left(x_{i}, b_{i}^{*} b_{j}, x_{j}\right) \geq 0$ in $A$, for all $x_{i} \in X, b_{i} \in B, i=1,2, \ldots, n$. It is clear that a positive definite map is weakly positive definite. Of course, the converse is not true in general: for example, if $A$ and $B$ are $\mathrm{C}^{*}$-algebras for which there exists a positive linear map $r_{0}: B \rightarrow A$ that is not completely positive, then the map $r: \mathbb{C} \times B \times \mathbb{C} \rightarrow A$ given by $r(\lambda, b, \mu)=\bar{\lambda} \mu r_{0}(b)$ is weakly positive definite but not positive definite.

The following definition is basic for the subsequent development.

Definition 2.1. Let $A$ and $B$ be unital $\mathrm{C}^{*}$-algebras and $X$ a TRO. A normalising context for $A, B$ and $X$ is a 5-tuple $(A, B, X, p, q)$, where

$$
p: X \times B \times X \rightarrow A
$$

and

$$
q: X \times A \times X \rightarrow B
$$

are weakly positive definite sesquilinear maps, $p$ (resp. $q$ ) is conjugate linear on the first (resp. third) variable, $p(x, 1, x) \neq 0$ for each non-zero $x \in X$, and the following identities hold, for all $x, x_{1}, x_{2}, y_{1}, y_{2} \in X, a, a_{1}, a_{2} \in A$ and $b, b_{1}, b_{2} \in B$ :

(a) $p\left(x_{1}, b_{1} q\left(y_{1}, a, y_{2}\right) b_{2}, x_{2}\right)=p\left(x_{1}, b_{1}, y_{1}\right) a p\left(y_{2}, b_{2}, x_{2}\right)$;

(a') $q\left(y_{1}, a_{1} p\left(x_{1}, b, x_{2}\right) a_{2}, y_{2}\right)=q\left(y_{1}, a_{1}, x_{1}\right) b q\left(x_{2}, a_{2}, y_{2}\right)$;

(b) $p\left(x, b, x_{1} x_{2}^{*} x_{3}\right)=p\left(x, b, x_{1}\right) p\left(x_{2}, 1, x_{3}\right)$.

A semi-normalising context for $A, B$ and $X$ is a 4-tuple denoted by $(A, B, X, p)$, where $p: X \times B \times X \rightarrow A$ is a positive definite sesquilinear map, conjugate linear on the first variable, such that $p(x, 1, x) \neq 0$ for each non-zero $x \in X$ and condition (b) is satisfied.

The motivation for the definition above comes from the following example.

ExAmple. Let $\mathcal{H}_{1}$ and $\mathcal{H}_{2}$ be Hilbert spaces, $\mathcal{A} \subseteq \mathcal{B}\left(\mathcal{H}_{1}\right), \mathcal{B} \subseteq \mathcal{B}\left(\mathcal{H}_{2}\right)$ be unital $\mathrm{C}^{*}$-algebras and $\mathcal{X} \subseteq \mathcal{B}\left(\mathcal{H}_{1}, \mathcal{H}_{2}\right)$ be a TRO, consisting of semi-normalisers of $\mathcal{B}$ into $\mathcal{A}$. Define a mapping $p: \mathcal{X} \times \mathcal{B} \times \mathcal{X} \rightarrow \mathcal{A}$ by $p(T, V, S)=T^{*} V S, T, S \in \mathcal{X}, V \in \mathcal{B}$. Then $(\mathcal{A}, \mathcal{B}, \mathcal{X}, p)$ is a semi-normalising context. If $\mathcal{X}$ normalises $\mathcal{B}$ into $\mathcal{A}$ and a mapping $q: \mathcal{X} \times \mathcal{A} \times \mathcal{X} \rightarrow \mathcal{B}$ is defined by $q(T, U, S)=T U S^{*}, T, S \in \mathcal{X}, U \in \mathcal{A}$, then $(\mathcal{A}, \mathcal{B}, \mathcal{X}, p, q)$ is a normalising context. One of our main aims in this note is to show that every (semi-) normalising context arises essentially in this way.

A standard argument yields the following fact.

Lemma 2.2. If $r: X \times B \times X \rightarrow A$ is a sesquilinear weakly positive definite map, then $r(x, b, y)^{*}=\left(y, b^{*}, x\right)$, for all $x, y \in X$ and $b \in B$.

As we pointed out, weak positive definiteness is strictly weaker than positive definiteness. As the next lemma shows, the two notions are the same for the maps of a normalising context. 
LeMmA 2.3. If $(A, B, X, p, q)$ is a normalising context, then $p$ and $q$ are positive definite.

Proof. By symmetry, it suffices to prove the claim for the map $p$. Let

$$
A_{0}=\operatorname{span}\left\{\sum_{i=1}^{n} p\left(x_{i}, b_{i}, y_{i}\right): x_{i}, y_{i} \in X, b_{i} \in B, i=1,2, \ldots, n, n \in \mathbb{N}\right\}^{-\|\cdot\|} .
$$

By condition (a) of Definition 2.1, the space $A_{0}$ is closed under multiplication and by Lemma 2.2, $A_{0}$ is selfadjoint. Hence $A_{0}$ is the $\mathrm{C}^{*}$-subalgebra of $A$ generated by the range of the map $p$. Fix elements $x_{i} \in X$ and $b_{i} \in B, i=1,2, \ldots, n$ and pick $a_{i}=\sum_{k=1}^{m_{i}} p\left(u_{i k}, b_{i k}, v_{i k}\right) \in A_{0}$. Then, by (a) and Lemma 2.2,

$$
\begin{aligned}
a_{0} & \stackrel{\text { def }}{=} \sum_{i, j=1}^{n} a_{i}^{*} p\left(x_{i}, b_{i}^{*} b_{j}, x_{j}\right) a_{j} \\
& =\sum_{i, j, k, l} p\left(u_{i k}, b_{i k}, v_{i k}\right)^{*} p\left(x_{i}, b_{i}^{*} b_{j}, x_{j}\right) p\left(u_{j l}, b_{j l}, v_{j l}\right) \\
& =\sum_{i, j, k, l} p\left(v_{i k}, b_{i k}^{*}, u_{i k}\right) p\left(x_{i}, b_{i}^{*} b_{j}, x_{j}\right) p\left(u_{j l}, b_{j l}, v_{j l}\right) \\
& =\sum_{i, j, k, l} p\left(v_{i k}, b_{i k}^{*} q\left(u_{i k}, 1, x_{i}\right) b_{i}^{*} b_{j} q\left(x_{j}, 1, u_{j l}\right) b_{j l}, v_{j l}\right) \\
& =\sum_{i, j, k, l} p\left(v_{i k}, c_{i k}^{*} c_{j l}, v_{j l}\right)
\end{aligned}
$$

where $c_{i k}=b_{i} q\left(x_{i}, 1, u_{i k}\right) b_{i k}, i=1,2, \ldots, n, k=1,2, \ldots, m_{i}$. It follows that $a_{0} \geq$ 0 in $A_{0}$; by Proposition 6.1 of [13], $\left(p\left(x_{i}, b_{i}^{*} b_{j}, x_{j}\right)\right)_{i j} \geq 0$ in $M_{n}\left(A_{0}\right)$, and thus $\left(p\left(x_{i}, b_{i}^{*} b_{j}, x_{j}\right)\right)_{i j} \geq 0$ in $M_{n}(A)$.

The next theorem provides an abstract characterisation of the normalising TRO's of (unital) $\mathrm{C}^{*}$-algebras: it yields that a TRO $X$ possesses a completely isometric representation consisting of normalisers between some faithful ${ }^{*}$-representations of $B$ and $A$ if and only if there exists a normalising context for $A, B$ and $X$.

TheOrem 2.4. Let $A$ and $B$ be unital $C^{*}$-algebras, $X$ a TRO and $p: X \times B \times X \rightarrow A$ (resp. $q: X \times A \times X \rightarrow B$ ) a sesquilinear map, conjugate linear on the first (resp. third) variable. The 5-tuple $(A, B, X, p, q)$ is a normalising context if and only if there exist Hilbert spaces $\mathcal{H}_{1}$ and $\mathcal{H}_{2}$, faithful ${ }^{*}$-representations $\pi_{1}: A \rightarrow \mathcal{B}\left(\mathcal{H}_{1}\right)$ and $\pi_{2}: B \rightarrow$ $\mathcal{B}\left(\mathcal{H}_{2}\right)$ and a ternary monomorphism $\varphi: X \rightarrow \mathcal{B}\left(\mathcal{H}_{1}, \mathcal{H}_{2}\right)$ such that

$$
\pi_{1}(p(x, b, y))=\varphi(x)^{*} \pi_{2}(b) \varphi(y) \quad \text { and } \quad \pi_{2}(q(x, a, y))=\varphi(x) \pi_{1}(a) \varphi(y)^{*},
$$

for all $x, y \in X, a \in A$ and $b \in B$.

Proof. Suppose that $(A, B, X, p, q)$ is a normalising context and let $Y=B \otimes X \otimes A$ be the algebraic tensor product of $B, X$ and $A$. Define pairings $(\cdot, \cdot)_{A}: Y \times Y \rightarrow A$ and $(\cdot, \cdot)_{B}: Y \times Y \rightarrow B$ given on elementary tensors by

$$
\left(b_{1} \otimes x_{1} \otimes a_{1}, b_{2} \otimes x_{2} \otimes a_{2}\right)_{A}=a_{1}^{*} p\left(x_{1}, b_{1}^{*} b_{2}, x_{2}\right) a_{2}
$$


and

$$
\left(b_{1} \otimes x_{1} \otimes a_{1}, b_{2} \otimes x_{2} \otimes a_{2}\right)_{B}=b_{1} q\left(x_{1}, a_{1} a_{2}^{*}, x_{2}\right) b_{2}^{*}
$$

If $\alpha=\sum_{i=1}^{n} b_{i} \otimes x_{i} \otimes a_{i} \in Y$ then, by Lemma 2.3,

$$
(\alpha, \alpha)_{A}=\sum_{i, j=1}^{n} a_{i}^{*} p\left(x_{i}, b_{i}^{*} b_{j}, x_{j}\right) a_{j} \geq 0 .
$$

Similarly, $(\alpha, \alpha)_{B} \geq 0$ for each $\alpha \in Y$.

For an element $\alpha \in Y$, set $\|\alpha\|_{A}=\sqrt{\left\|(\alpha, \alpha)_{A}\right\|}$ and $\|\alpha\|_{B}=\sqrt{\left\|(\alpha, \alpha)_{B}\right\|}$ and let $N_{1}$ (resp. $N_{2}$ ) be the kernel of $\|\cdot\|_{A}$ (resp. $\|\cdot\|_{B}$ ). Suppose that $\alpha=\sum_{i=1}^{n} b_{i} \otimes x_{i} \otimes a_{i} \in N_{2}$. Then $\sum_{i, j=1}^{n} b_{i} q\left(x_{i}, a_{i} a_{j}^{*}, x_{j}\right) b_{j}^{*}=0$. Thus

$$
\sum_{i, j, k, l=1}^{n} a_{k}^{*} p\left(x_{k}, b_{k}^{*} b_{i} q\left(x_{i}, a_{i} a_{j}^{*}, x_{j}\right) b_{j}^{*} b_{l}, x_{l}\right) a_{l}=0 .
$$

By condition (a) of Definition 2.1,

$$
\left(\sum_{k, i} a_{k}^{*} p\left(x_{k}, b_{k}^{*} b_{i}, x_{i}\right) a_{i}\right)\left(\sum_{k, i} a_{k}^{*} p\left(x_{k}, b_{k}^{*} b_{i}, x_{i}\right) a_{i}\right)^{*}=0
$$

and so $\sum_{k, i} a_{k}^{*} p\left(x_{k}, b_{k}^{*} b_{i}, x_{i}\right) a_{i}=0$, which means that $\sum_{i=1}^{n} b_{i} \otimes x_{i} \otimes a_{i} \in N_{1}$. Hence $N_{2} \subseteq N_{1}$ and, by symmetry, $N_{1} \subseteq N_{2}$; let $N=N_{1}=N_{2}$.

If $\alpha \in N$ and $\beta \in Y$, the Cauchy-Schwarz inequality implies that $(\alpha, \beta)_{A}=0$ and $(\alpha, \beta)_{B}=0$. Thus $(\cdot, \cdot)_{A}$ and $(\cdot, \cdot)_{B}$ give rise to $A$-valued and $B$-valued pairings on the factor space $Z_{0} \stackrel{\text { def }}{=} Y / N$, which we denote in the same way and which are defined by $(\alpha+N, \beta+N)_{A}=(\alpha, \beta)_{A}$ and $(\alpha+N, \beta+N)_{B}=(\alpha, \beta)_{B}$.

Define a right $A$ - and a left $B$-module actions on $Y$ by setting

$$
(b \otimes x \otimes a) \cdot a_{0}=b \otimes x \otimes a a_{0}
$$

and

$$
b_{0} \cdot(b \otimes x \otimes a)=b_{0} b \otimes x \otimes a .
$$

It is clear that these are indeed module operations. Next observe that if we let $\alpha=\sum_{i=1}^{n} b_{i} \otimes x_{i} \otimes a_{i} \in N$ and $a_{0} \in A$ then

$$
\left(\alpha \cdot a_{0}, \alpha \cdot a_{0}\right)_{A}=\sum_{i, j=1}^{n} a_{0}^{*} a_{i}^{*} p\left(x_{i}, b_{i}^{*} b_{j}, x_{j}\right) a_{j} a_{0}=0
$$

and hence $N \cdot A \subseteq N$; similarly, $B \cdot N \subseteq N$. Equip $Z_{0}$ with the induced module actions.

We now show that $Z_{0}$ is a pre-Hilbert $B, A$-bimodule. The identities $\left(z_{1}, z_{2}\right)_{A}^{*}=$ $\left(z_{2}, z_{1}\right)_{A}$ and $\left(z_{1}, z_{2}\right)_{B}^{*}=\left(z_{2}, z_{1}\right)_{B}$ follow from Lemma 2.2. Positive definiteness has already been observed. The relations $\left(z_{2}, z_{1} \cdot a\right)_{A}=\left(z_{2}, z_{1}\right)_{A} a$ and $\left(b \cdot z_{2}, z_{1}\right)_{B}=$ $b\left(z_{2}, z_{1}\right)_{B}$ follow directly from the definition of the pairings and the module operations. 
Let $\alpha_{i}=b_{i} \otimes x_{i} \otimes a_{i} \in Y, i=1,2,3$; we compute in $Y$ :

$$
\begin{aligned}
\beta \stackrel{\text { def }}{=} & \left(\alpha_{1}, \alpha_{2}\right)_{B} \cdot \alpha_{3}-\alpha_{1} \cdot\left(\alpha_{2}, \alpha_{3}\right)_{A} \\
= & \left(b_{1} \otimes x_{1} \otimes a_{1}, b_{2} \otimes x_{2} \otimes a_{2}\right)_{B} \cdot\left(b_{3} \otimes x_{3} \otimes a_{3}\right) \\
& -\left(b_{1} \otimes x_{1} \otimes a_{1}\right) \cdot\left(b_{2} \otimes x_{2} \otimes a_{2}, b_{3} \otimes x_{3} \otimes a_{3}\right)_{A} \\
= & \left(b_{1} q\left(x_{1}, a_{1} a_{2}^{*}, x_{2}\right) b_{2}^{*} b_{3}\right) \otimes x_{3} \otimes a_{3}-b_{1} \otimes x_{1} \otimes\left(a_{1} a_{2}^{*} p\left(x_{2}, b_{2}^{*} b_{3}, x_{3}\right) a_{3}\right) .
\end{aligned}
$$

If $d \otimes y \otimes c \in Y$ is an elementary tensor then, by (a),

$$
\begin{aligned}
(d \otimes y \otimes c, \beta)_{A}= & c^{*} p\left(y, d^{*} b_{1} q\left(x_{1}, a_{1} a_{2}^{*}, x_{2}\right) b_{2}^{*} b_{3}, x_{3}\right) a_{3} \\
& -c^{*} p\left(y, d^{*} b_{1}, x_{1}\right) a_{1} a_{2}^{*} p\left(x_{2}, b_{2}^{*} b_{3}, x_{3}\right) a_{3}=0 .
\end{aligned}
$$

It follows that $\|\beta\|_{A}=0, \beta \in N$ and, by (conjugate-)linearity, $\left(z_{1}, z_{2}\right)_{B} \cdot z_{3}=$ $z_{1} \cdot\left(z_{2}, z_{3}\right)_{A}$, for all $z_{1}, z_{2}, z_{3} \in Z_{0}$.

We have thus shown that $Z_{0}$ is a pre-Hilbert $B, A$-bimodule; let $Z$ be its completion. Recall that the canonical ternary structure of $Z$ is given by $z_{1} z_{2}^{*} z_{3}=z_{1} \cdot\left(z_{2}, z_{3}\right)_{A}$. Consider the map $\iota: X \rightarrow Z$, defined by

$$
\iota(x)=1 \otimes x \otimes 1+N \quad(x \in X) .
$$

We have $\|\iota(x)\|^{2}=\|p(x, 1, x)\|>0$ and thus $\iota$ is injective. Let

$$
\gamma_{1} \stackrel{\text { def }}{=} \iota\left(x_{1} x_{2}^{*} x_{3}\right)=1 \otimes\left(x_{1} x_{2}^{*} x_{3}\right) \otimes 1+N
$$

We have

$$
\begin{aligned}
\gamma_{2} & \stackrel{\text { def }}{=} \iota\left(x_{1}\right) \iota\left(x_{2}\right)^{*} \iota\left(x_{3}\right) \\
& =\left(1 \otimes x_{1} \otimes 1+N\right)\left(1 \otimes x_{2} \otimes 1+N\right)^{*}\left(1 \otimes x_{3} \otimes 1+N\right) \\
& =\left(1 \otimes x_{1} \otimes 1+N\right) \cdot\left(1 \otimes x_{2} \otimes 1+N, 1 \otimes x_{3} \otimes 1+N\right)_{A} \\
& =1 \otimes x_{1} \otimes p\left(x_{2}, 1, x_{3}\right)+N .
\end{aligned}
$$

If $d \otimes y \otimes c+N \in Z$ then, by (b),

$$
\left(d \otimes y \otimes c+N, \gamma_{1}-\gamma_{2}\right)_{A}=c^{*} p\left(y, d, x_{1} x_{2}^{*} x_{3}\right)-c^{*} p\left(y, d, x_{1}\right) p\left(x_{2}, 1, x_{3}\right)=0 .
$$

It follows that $\gamma_{1}-\gamma_{2} \in N$; hence $\iota$ is a ternary monomorphism from $X$ into $Z$.

We have thus constructed a Hilbert $B, A$-bimodule $\left(Z,(\cdot, \cdot)_{A},(\cdot, \cdot)_{B}\right)$ and a ternary monomorphism $\iota: X \rightarrow Z$ such that

$$
p(x, b, y)=(\iota(x), b \cdot \iota(y))_{A} \quad \text { and } \quad q(x, a, y)=(\iota(x) \cdot a, \iota(y))_{B},
$$

for all $x, y \in X, a \in A$ and $b \in B$. By Proposition 2.3 of [4], we can form the linking $C^{*}$-algebra of $Z$ consisting of all matrices of the form $\left(\begin{array}{cc}b & x \\ \tilde{y} & a\end{array}\right)$, where $a \in A, b \in B$, $x \in Z$ and $\tilde{y} \in \tilde{Z}$ ( $\tilde{Z}$ being the "dual" $A, B$-bimodule to $Z$ [4]); the involution and the multiplication are performed in the obvious way using the module operations and the pairings. A standard argument now implies that there exist Hilbert spaces $\mathcal{H}_{1}$ and $\mathcal{H}_{2}$, faithful ${ }^{*}$-representations $\pi_{1}: A \rightarrow \mathcal{B}\left(\mathcal{H}_{1}\right)$ and $\pi_{2}: B \rightarrow \mathcal{B}\left(\mathcal{H}_{2}\right)$ and a ternary monomorphism $\psi: Z \rightarrow \mathcal{B}\left(\mathcal{H}_{1}, \mathcal{H}_{2}\right)$ such that $\psi(b \cdot x \cdot a)=\pi_{2}(b) \psi(x) \pi_{1}(a)$, $\pi_{1}\left((x, y)_{A}\right)=\psi(x)^{*} \psi(y)$ and $\pi_{2}\left((x, y)_{B}\right)=\psi(x) \psi(y)^{*}$, for all $x, y \in Z, a \in A$ and $b \in B$. The claim follows if we set $\varphi=\psi \circ \iota$. 
Conversely, suppose that representations $\pi_{1}, \pi_{2}$ and $\varphi$ exist with the cited properties. Clearly, $p$ and $q$ are weakly positive definite. Conditions (a), (a') and (b) follow from the associativity of the operator multiplication. By the $\mathrm{C}^{*}$-property, $\|p(x, 1, x)\|=\|x\|^{2}>0$ and hence $p(x, 1, x) \neq 0$, for each $x \in X$.

Theorem 2.4 yields the following characterisation of the $\mathrm{C}^{*}$-algebras possessing non-trivial ideals that are strongly Morita equivalent in the sense in Rieffel [16].

Corollary 2.5. Let $A$ and $B$ be unital $C^{*}$-algebras. If $(A, B, X, p, q)$ is a normalising context for $A$ and $B$, then the ideals of $A$ and $B$ generated by the ranges of $p$ and $q$, respectively, are strongly Morita equivalent. Hence, $A$ and $B$ have non-trivial strongly Morita equivalent ideals if and only if $A$ and $B$ participate in some normalising context.

REMARK. By Theorem 2.4, for a normalising context $(A, B, X, p, q)$, the identity "dual" to (b), $q\left(y, a, y_{1} y_{2}^{*} y_{3}\right)=q\left(y, a, y_{3}\right) q\left(y_{2}, 1, y_{1}\right)$, is satisfied automatically. Also automatic are the identities $\|p(x, 1, x)\|=\|x\|^{2}$ and $\|q(x, 1, x)\|=\|x\|^{2},(x \in X)$. By Lemma 2.3 we now have that if $(A, B, X, p, q)$ is a normalising context then $(A, B, X, p)$ and $\left(B, A, X^{*}, q^{*}\right)$ are semi-normalising contexts, where $X^{*}$ is the TRO consisting of the adjoints of the elements of $X$ and $q^{*}: X^{*} \times A \times X^{*} \rightarrow B$ is the map given by $q^{*}\left(x^{*}, a, y^{*}\right)=q(x, a, y)$.

We now turn our attention to semi-normalisers between $C^{*}$-algebras. Recall that a right-Hilbert $B, A$-bimodule is a right Hilbert $A$-module $Z$ equipped with a left $B$ module action induced by a unital *-homomorphism $B \rightarrow \mathcal{B}(Z)$, where $\mathcal{B}(Z)$ is the $\mathrm{C}^{*}$-algebra of adjointable operators on $X$. (For definitions and basic facts concerning operators on Hilbert modules see [10].) The next result is the analogue of Theorem 2.4 in the semi-normalisers case. It provides a characterisation of the TRO's which can be completely isometrically represented as a space of semi-normalisers between some *-representations of two given $\mathrm{C}^{*}$-algebras.

THEOREM 2.6. Let $A$ and $B$ be unital $C^{*}$-algebras, $X$ be a TRO and $p: X \times B \times X \rightarrow$ $A$ a sesquilinear positive definite map, conjugate linear on the first variable. The 4-tuple $(A, B, X, p)$ is a semi-normalising context if and only if there exist Hilbert spaces $\mathcal{H}_{1}$ and $\mathcal{H}_{2}$, a faithful ${ }^{*}$-representation $\pi_{1}: A \rightarrow \mathcal{B}\left(\mathcal{H}_{1}\right)$, a non-degenerate ${ }^{*}$-representation $\pi_{2}: B \rightarrow \mathcal{B}\left(\mathcal{H}_{2}\right)$ and a ternary monomorphism $\varphi: X \rightarrow \mathcal{B}\left(\mathcal{H}_{1}, \mathcal{H}_{2}\right)$ such that

$$
\pi_{1}(p(x, b, y))=\varphi(x)^{*} \pi_{2}(b) \varphi(y),
$$

for all $x, y \in X$ and $b \in B$.

Proof. Assume that $(A, B, X, p)$ is a semi-normalising context. As in the proof of Theorem 2.4, equip the (algebraic) tensor product $Y=B \otimes X \otimes A$ with an $A$-valued sesquilinear form given on elementary tensors by

$$
\left(b_{1} \otimes x_{1} \otimes a_{1}, b_{2} \otimes x_{2} \otimes a_{2}\right)_{A}=a_{1}^{*} p\left(x_{1}, b_{1}^{*} b_{2}, x_{2}\right) a_{2} .
$$

Let $N$ be the kernel of the induced seminorm $\|\cdot\|_{A}$ and consider the module operation on $Y$ given by $(b \otimes x \otimes a) \cdot a_{0}=b \otimes x \otimes a a_{0}$. As before, observe that $Z_{0}=Y / N$ is a right pre-Hilbert $A$-module, let $Z$ be its completion and notice that the map $\iota: X \rightarrow Z$ given by $\iota(x)=1 \otimes x \otimes 1+N$ is a ternary monomorphism.

Let $\mathcal{K}(Z)$ and $\mathcal{B}(Z)$ be the $\mathrm{C}^{*}$-algebras of compact and adjointable operators on $Z$, respectively, and $\mathcal{L}(Z)$ the Banach algebra of bounded $A$-module linear maps on $Z$. 
(See [10].) For each $b_{0} \in B$, define a linear map $\pi\left(b_{0}\right)$ on $Y$ given on elementary tensors by

$$
\pi\left(b_{0}\right)(b \otimes x \otimes a)=b_{0} b \otimes x \otimes a .
$$

The positive definiteness of $p$ implies that $\pi\left(b_{0}\right) N \subseteq N$ and $\left\|\pi\left(b_{0}\right)(\alpha)\right\|_{A} \leq\left\|b_{0}\right\|\|\alpha\|_{A}$, for all $b_{0} \in B$ and $\alpha \in Z$. Thus $\pi$ induces a contractive homomorphism from $B$ into $\mathcal{L}(Z)$. If $\alpha=\sum_{i=1}^{n} b_{i} \otimes x_{i} \otimes a_{i}, \beta=\sum_{j=1}^{m} d_{j} \otimes y_{j} \otimes c_{j}$, then

$$
\begin{aligned}
\left(\pi\left(b_{0}\right)(\alpha+N), \beta+N\right)_{A} & =\left(\sum_{i=1}^{n} b_{0} b_{i} \otimes x_{i} \otimes a_{i}, \sum_{j=1}^{m} d_{j} \otimes y_{j} \otimes c_{j}\right)_{A} \\
& =\sum_{i, j} a_{i}^{*} p\left(x_{i}, b_{i}^{*} b_{0}^{*} d_{j}, y_{j}\right) c_{j} \\
& =\left(\sum_{i=1}^{n} b_{i} \otimes x_{i} \otimes a_{i}, \sum_{j=1}^{m} b_{0}^{*} d_{j} \otimes y_{j} \otimes c_{j}\right)_{A} \\
& =\left(\alpha+N, \pi\left(b_{0}^{*}\right)(\beta+N)\right)_{A} .
\end{aligned}
$$

Hence $\pi\left(b_{0}\right) \in \mathcal{B}(Z)$ and $\pi$ is a (unital) *-homomorphism. We have thus constructed a right-Hilbert $B, A$-bimodule $\left(Z,(\cdot, \cdot)_{A}\right)$ and a ternary monomorphism $\iota: X \rightarrow Z$ such that $p(x, b, y)=(\iota(x), b \cdot \iota(y))_{A}$, for all $x, y \in X$ and $b \in B$. By Theorem 3.1 of [11], there exist Hilbert spaces $\mathcal{H}_{1}$ and $\mathcal{H}_{2}$, a faithful ${ }^{*}$-representation $\pi_{1}: A \rightarrow \mathcal{B}\left(\mathcal{H}_{1}\right)$ and a ternary monomorphism $\psi: Z \rightarrow \mathcal{B}\left(\mathcal{H}_{1}, \mathcal{H}_{2}\right)$ such that $\pi_{1}\left(\left(z_{1}, z_{2}\right)\right)=\psi\left(z_{1}\right)^{*} \psi\left(z_{2}\right)$ and $\psi(z \cdot a)=\psi(z) \pi_{1}(a), z, z_{1}, z_{2} \in Z, a \in A$. The closed linear span $\mathcal{C}$ of $\psi(Z) \psi(Z)^{*}$ is then a $C^{*}$-algebra on $\mathcal{H}_{2},{ }^{*}$-isomorphic to $\mathcal{K}(Z)$. See e.g. [1, pp. 289-290]. The space $\mathcal{H}_{2}$ can moreover be taken in such a way that $\mathcal{C}$ is nondegenerate. A well-known result of Kasparov (Theorem 2.4 of [11]) identifies $\mathcal{B}(Z)$ as the multiplier algebra of $\mathcal{K}(Z)$. Thus there exists a ${ }^{*}$-isomorphism $\pi_{0}$ of $\mathcal{B}(Z)$ onto the multiplier algebra $\mathcal{D} \subseteq \mathcal{B}\left(\mathcal{H}_{2}\right)$ of $\mathcal{C}$ computed in $\mathcal{B}\left(\mathcal{H}_{2}\right)$. Letting $\pi_{2}=\pi_{0} \circ \pi$ and $\varphi=\psi \circ \iota$ we see that $\pi_{1}(p(x, b, y))=\varphi(x)^{*} \pi_{2}(b) \varphi(y), x, y \in X, b \in B$. The proof is complete.

We note that versions of Theorems 2.4 and 2.6 can be proved without the assumption that the $C^{*}$-algebras $A$ and $B$ are unital - one can define "non-unital (semi-)normalising contexts" replacing the terms in which the units appear by limits along approximate identities. (A version of Corollary 2.5 will then be valid without the assumption that $A$ and $B$ are unital.) We hence have the corollaries of Theorem 2.6 that follow. The first one is a representation for positive definite maps. In the case $B=\mathbb{C}$, it can also be inferred from Theorem 2.3 of [11].

Corollary 2.7. Let $A$ and $B$ be $C^{*}$-algebras and $X$ a linear space over the complex field. A sesquilinear map $p: X \times B \times X \rightarrow A$, conjugate linear on the first variable, is positive definite if and only if there exists a right-Hilbert $B, A$-bimodule $Z$ and a linear map $j: X \rightarrow Z$ such that $p(x, b, y)=(j(x), b \cdot j(y))_{A}$, for all $x, y \in X$ and $b \in B$.

We also have the following characterisation of the TRO's, ternarily isomorphic to a (ternary) subspace of some Hilbert $A$-module. Note that such subspaces are not necessarily $A$-submodules. 
Corollary 2.8. Let $A$ be a $C^{*}$-algebra. A TRO X is ternarily isomorphic to a subspace of a Hilbert $A$-module if and only if there exists a sesquilinear map $p: X \times X \rightarrow A$, conjugate linear on the first variable, such that $p(x, x)$ is a non-zero positive element of $A$, for each non-zero $x \in X$, and

$$
p\left(x, y_{1} y_{2}^{*} y_{3}\right)=p\left(x, y_{1}\right) p\left(y_{2}, y_{3}\right),
$$

for all $x, y_{1}, y_{2}, y_{3} \in X$.

We finally note that weak* versions of Theorems 2.4 and 2.6 can be provided. Recall that a $W^{*}-T R O$ is a TRO $X$ that is a dual Banach space [17]. If $A$ and $B$ are $\mathrm{W}^{*}$-algebras, a $W^{*}$-normalising context $(A, B, X, p, q)$ can be defined in the same way as in Definition 2.1, but with the additional requirement that the maps $p$ and $q$ are weak* continuous on each variable. The proof of the next result is based on the proof of Theorem 2.4 and uses the notion of a self-dual Hilbert module; we omit it. A similar result holds for semi-normalisers.

TheOREM 2.9. Let $A$ and $B$ be $W^{*}$-algebras and $X$ a $W^{*}$-TRO. There exists a $W^{*}$-normalising context for $A, B$ and $X$ if and only if there exist Hilbert spaces $\mathcal{H}_{1}$ and $\mathcal{H}_{2}$, faithful normal ${ }^{*}$-representations $\pi_{1}: A \rightarrow \mathcal{B}\left(\mathcal{H}_{1}\right)$ and $\pi_{2}: B \rightarrow \mathcal{B}\left(\mathcal{H}_{2}\right)$ and a ternary monomorphism $\varphi: X \rightarrow \mathcal{B}\left(\mathcal{H}_{1}, \mathcal{H}_{2}\right)$ such that $\varphi(X)$ is weakly closed and consists of normalisers of $\pi_{2}(B)$ into $\pi_{1}(A)$.

ACKNOWLEDGEMENT. The author wishes to thank the referee for his/her useful comments.

\section{REFERENCES}

1. D. P. Blecher, The Shilov boundary of an operator space and the characterization theorems, J. Functional Analysis 182 (2001), 280-343.

2. D. P. Blecher, On Morita's fundamental theorem for $\mathrm{C}^{*}$-algebras, Math. Scand $\mathbf{8 8}$ (2001), 137-153.

3. D. P. Blecher, P. S. Muhly and V. I. Paulsen, Categories of operator modules (Morita equivalence and projective modules), Mem. Amer. Math. Soc., 143 (2000), no. 681.

4. L. G. Brown, J. A. Mingo and N.-T. Shen, Quasi-multiplisers and embeddings of Hilbert C*-modules, Canadian J. Math. 46 (6) (1994), 1150-1174.

5. K. J. Coates, Normalizers of nest algebras, Proc. Amer. Math. Soc. 126 (1998), 159-165; Errata, ibid, 2511-2512.

6. E. Effros and Z. J. Ruan, Operator spaces (Clarendon Press, Oxford, 2000).

7. M. Hamana, Triple envelopes and Shilov boundaries of operator spaces, Math. J. Toyama Univ. 22 (1999), 77-93.

8. A. Katavolos and I.G. Todorov, Normalizers of operator algebras and reflexivity, Proc. London Math. Soc. (3) 86 (2003), 463-484.

9. M. Kaur and Z.-J. Ruan, Local properties of ternary rings of operators and their linking $\mathrm{C}^{*}$-algebras, J. Functional Analysis 195 (2002), 265-305.

10. E. C. Lance, Hilbert $C^{*}$-modules. A toolkit for operator algebraists (Cambridge University Press, 1995).

11. G. J. Murphy, Positive definite kernels and Hilbert C*-modules, Proc. Edinburgh Math. Soc. (2) 40 (1997), 367-374.

12. F. G. Murray and J. von Neumann, On rings of operators, Ann. of Math. 37 (1936), $116-229$.

13. W. L. Paschke, Inner product modules over B*-algebras, Trans. Amer. Math. Soc. 182 (1973), 443-568. 
14. W. L. Paschke, Induced representations of $C^{*}$-algebras, Adv. Math. 13 (1974), 176-257.

15. S. C. Power, Limit algebras - an introduction to subalgebras of $C^{*}$-algebras (Pitman Research Notes in Mathematics Studies No 278, 1992).

16. M. Rieffel, Morita equivalence for $\mathrm{C}^{*}$-algebras and $\mathrm{W}^{*}$-algebras, J. Pure Appl. Algebra 5 (1974), 51-96.

17. Zh.-J. Ruan, Type decomposition and rectangular AFD property for W*-TRO's (2002), preprint.

18. I. G. Todorov, A characterization of Morita equivalence pairs, Glasgow Math. J. 44 (2002), 535-545.

19. H. Zettl, A characterization of ternary rings of operators, Adv. Math. 48 (1983), 117143. 